\title{
Bourdieu y el pragmatismo norteamericano acerca de la creatividad del hábito1
}

\author{
Bourdieu and the American pragmatism on the habit's creativity
}

\section{Louis Quéré*}

* Director Emérito en el Centre National de la Recherche Scientifique -

Miembro del Instituto Marcel Mauss (Centro de Estudios de Movimientos sociales, CEMS) École des Hautes Études en Sciences Sociales, Francia I quere@ehess.fr

Traducción de Aimé Pansera

\section{PALABRAS CLAVE}

Disposición

Entorno

Hábito

Habitus

Impulso

Rutina

Savoir-faire

Esquema

Estructura

\section{KEYWORDS}

Disposition

Environment

Habit

Habitus

Impulse

Routine

Expertise

Scheme

Structure

\section{RESUMEN}

Varios autores insistieron en la cercana afinidad entre las concepciones de Bourdieu y las del pragmatismo norteamericano, sobre todo en el análisis del hábito. Emanuel Bourdieu relacionó el disposicionalismo de su padre con el de Charles S. Peirce. Privilegiaré aquí a otro autor de la tradición pragmatista, J. Dewey, que no es disposicionalista sino "actualista”. Hay numerosas similitudes entre el concepto bourdieusiano de habitus y la concepción deweyana del habit. Pero también hay diferencias importantes, y son sobre todo éstas las que quiero resaltar.

\section{ABSTRACT}

Several authors have highlighted the close affinity between Pierre Bourdieu's conceptions and those of American pragmatism, in particular on the analysis of habit. Emmanuel Bourdieu compared his father's dispositionalism with Charles S. Peirce's. I will focus on a different pragmatist author, namely John Dewey, who is an "actualist" - as opposed to a dispositionalist. There are many similarities between Bourdieu's concept of habitus and Dewey's concept of habit. Yet there are also important differences I would like to emphasize. 
Varios autores insistieron en la cercana afinidad entre las concepciones de Bourdieu y las del pragmatismo norteamericano, y hasta sugirieron que la obra de Bourdieu tiene plenamente "su lugar en el desarrollo en curso del pragmatismo” (Shusterman, 1995, p.603), y que permitiría mejorar las intuiciones de éste gracias a los conceptos más precisos, verificados empíricamente, que esta obra ofrece. El de habitus es uno de los más destacados por el lugar esencial que los pragmatistas le otorgaron al hábito. Sin embargo, hablaban de habit y no de habitus. Aunque Bourdieu fue lector de Peirce y un poco de Dewey, no es de ellos de quienes obtuvo la inspiración para elaborar su concepto de habitus, sino de Max Weber, en la fenomenología husserliana y poshusserliana (sobre todo en Merleau-Ponty con su problemática de la intencionalidad motriz-corporal), con la atención puesta en la gramática generativa de Chomsky, de moda en ese momento, no sin un cierto contrasentido: el habitus como generador de prácticas y de estrategias situadas está más ligado a la “creatividad del uso”, sobre la cual Chomsky no tenía nada para decir, que a la generatividad formal de su gramática, que es más bien de naturaleza mecánica (cf. Bouveresse, 2015).

El concepto de habitus proviene de la recuperación escolástica de conceptos aristotélicos, sobre todo el de hexis y el de eidos. El hexis es un estado habitual (principalmente del cuerpo) adquirido por la repetición de actos conformes a la razón, repetición que forma hábitos. Este estado habitual dirige el accionar en su conjunto; el que dispone de él tiene un sentido asegurado de lo que se debe o de lo que tiene que hacer. El habitus es a la vez, de este modo, una manera de ser, una disposición o una propensión, y un poder generador. Estas son dimensiones que Bourdieu conservó, y a su vez modificó (cf. Haber, 2004; Héran, 1987; Gautier, 2012).

Su concepto de habitus sigue siendo claramente disposicionalista. Bourdieu, que habla indistintamente de sistema de disposiciones y de sistema de esquemas (los habitus son uno y otro), asume esto totalmente frente a la crítica wittgensteiniana del concepto de disposición (Bourdieu, 1997, p.163). Explica que no se puede abandonar el concepto de disposición en socioantropología, excepto en el caso de que se niegue la existencia de aprendizajes: "Hablar de disposición, es simplemente tomar nota de una disposición natural de los cuerpos humanos (...) la condicionabilidad como capacidad natural de adquirir capacidades no naturales, arbitrarias” (Bourdieu, 1997, p.163)

Este disposicionalismo lo acerca más a Peirce, tal y como lo demostró espléndidamente Emmanuel Bourdieu, pero menos a James y a Dewey. Hay un “disposicionalismo pesado” en Peirce (Chauviré, 2002), que Dewey no comparte en absoluto y que está sin duda aligerado en Bourdieu. Para Peirce, saber un idioma, por ejemplo, es una disposición, es decir,

una potencialidad permanente, que se actualiza de tanto en tanto, en ocasiones precisas, y que hasta puede nunca actualizarse; no es por ello menos real, porque, según Peirce, los universales (leyes, hábitos, significaciones) son reales, en el sentido de que operan realmente en la naturaleza (Chauviré, 2002, p.28)

Sabemos que Peirce concebía también las creencias como hábitos de acción, es decir, como disposiciones para actuar de tal o cual manera en tal o cual circunstancia. Dewey desconfiaba de este “disposicionalismo duro”, por razones que voy a intentar explicitar aquí. Es este el pragmatismo que voy a privilegiar. 
El argumento que quiero desarrollar es que los proyectos de Dewey y de Bourdieu, ambos grandes lectores de antropología, pueden difícilmente encontrarse, aunque se superpongan en varios puntos: mientras que Dewey busca naturalizar y “ecologizar” el espíritu, la razón, el conocimiento y la acción, Bourdieu busca socializarlos e historizarlos, dentro de un marco de pensamiento que sigue siendo estructuralista. El modelo de Dewey es biológico y ecológico (porque es energético), y nutrido por una filosofía del acontecimiento; el de Bourdieu sociológico e histórico, y nutrido por una forma de estructuralismo. Además, Dewey no necesitaba conquistar, frente al estructuralismo, la idea de una creatividad del obrar, oponiéndose a explicaciones que la negasen o la desconociesen; ésta era evidente desde el comienzo, y su elucidación pasa a la vez por un análisis del hábito, por una consideración de la serialidad del comportamiento, por una reconceptualización de la dinámica medios-fines y de la formación de valores a la luz de una preeminencia otorgada a las consecuencias, y por una teoría de la investigación. Voy a rememorar primero el habitus de Bourdieu, antes de presentar más ampliamente el enfoque de Dewey, menos conocido.

\section{El “estructuralismo genético” de Bourdieu}

Bourdieu y Dewey no tienen, por lo tanto, las mismas preocupaciones; sobre todo, no hablan en el mismo contexto intelectual y cultural. Dewey escribe tras los pasos de Darwin y de James. Para James, quien escribió en 1887 el pequeño libro Habit, el hábito es "el enorme volante motor (flywheel) de la sociedad, su agente de conservación más preciado”; el capítulo 4 del volumen 1 de los Principles of psychology (1890) en donde figura esta cita (p. 121), está dedicado también al hábito (Kilpinen, 2000) y toma posición en contra de la psicología de su época, en la que predominan la psicología de la conciencia, la psicología del instinto y el behaviorismo mecanicista. Se esfuerza por restaurar una continuidad entre la naturaleza y el espíritu, rechazando las explicaciones clásicas de las capacidades específicas del hombre y las distinciones que las sustentan. Al modelo del arco reflejo también le opone un enfoque holístico del comportamiento, insistiendo en la secuencialidad y la serialidad de la conducta. Bourdieu comparte el antiintelectualismo de James y de Dewey (su definición es casi la misma que la de éstos); su crítica de la postura escolástica y su interés por el sentido práctico o la lógica de la práctica lo demuestran. Sin embargo, siendo sociólogo y antropólogo, busca sociologizar e historizar el espíritu y el comportamiento humanos, más que naturalizarlos. El habitus es efectivamente una historia social hecha cuerpo, a través de la formación de un sistema de disposiciones “durables y extrapolables”, que tienen una cierta inercia, justamente la de una estructura. Las normas, la razón, el universal, los instrumentos de conocimiento, los principios organizadores de la acción y más ampliamente las estructuras mentales o cognitivas (esquemas de percepción y de evaluación, esquemas de pensamiento, esquemas clasificatorios, sistemas de clasificación, etc.) son socialmente constituidos y hay que darles su profundidad histórica (con su porción de contingencia y arbitrariedad). Para él, la sociología tiene la obligación de ser al mismo tiempo estructural e histórica.

Bourdieu concibe su teoría del habitus en una confrontación, por un lado con la filosofía fenomenológico-existencialista en boga en los años 50 y 60 (Sartre y otros), y por el otro con el estructuralismo, el de Lévi-Strauss, con el marxismo estructuralista de Althusser o hasta con la 
lingüística estructural de Saussure (que concebía la práctica como simple ejecución). La primera es acusada de celebrar lo "vivido" y de ser incapaz de "dar cuenta de la necesidad del mundo social" (Bourdieu, 1980, p. 88), mientras que el estructuralismo lo es de adoptar un punto de vista superficial sobre el mundo social y de desconocer la relación al mundo social que es la de la experiencia cotidiana; es también acusado de abolir la creatividad de los agentes, quienes son concebidos como simples "soportes" o portadores de la estructura, o como "autómatas configurados como relojes” (Bourdieu, 1987, p. 19). Es a propósito que Bourdieu habla de agentes y no de sujetos; busca resaltar su agentividad (su capacidad de invención y de improvisación).

Pero, por otra parte, no reniega completamente del proyecto estructuralista, y conserva a menudo su vocabulario:

Intento elaborar un estructuralismo genético: el análisis de las estructuras objetivas (las de los diferentes campos) es inseparable del análisis de la génesis, en individuos biológicos, de las estructuras mentales, que son en parte el producto de la incorporación de las estructuras sociales y del análisis de estas mismas estructuras sociales² (Bourdieu, 1987, p. 24)

Bourdieu fue precedido, en este proyecto de estructuralismo genético, por Jean Piaget y Lucien Goldman.

El otro blanco de Bourdieu es la teoría de la elección racional y su finalismo. Los agentes, explica Bourdieu, no son “calculadores racionales”, y la lógica de sus prácticas no es la de una racionalidad teórica, sino un "sentido práctico": "Las conductas pueden estar orientadas en relación a fines sin estar conscientemente dirigidas hacia esos fines, dirigidas por esos fines” (Bourdieu, 1987, p.20). Los agentes no son tampoco seguidores de reglas: precisamente,

el sentido práctico es lo que permite accionar como «se debe» (...) sin dar ni ejecutar (...) una regla de conducta. (...) Los esquemas del habitus (...) permiten adaptarse sin cesar a contextos parcialmente modificados y deconstruir la situación como un conjunto dotado de sentido, en una operación práctica de anticipación cuasi corporal de las tendencias inmanentes del campo y de las conductas engendradas por todos los habitus isomorfos (...) con los cuales están en comunicación inmediata porque les son espontáneamente otorgados (Bourdieu, 1997, p.166-7).

\section{Dos grandes cuestiones}

La teoría del habitus pretende resolver simultáneamente varias cuestiones. Podemos agruparlas en dos grandes categorías. La primera tiene que ver con la naturaleza del sentido práctico, con la lógica de la práctica y la explicación de la regularidad de las conductas: ¿qué es lo que asegura la regularidad de las prácticas, si entendemos que no es "la obediencia consciente a reglas conscientemente elaboradas y sancionadas” (Bourdieu, 1980, p.67) y que la práctica es relativamente indeterminada? La segunda refiere a la reproducción social y al condicionamiento social, o mejor dicho a los determinantes sociales, históricamente constituidos, de las prácticas, las estructuras mentales y la subjetividad. Subyaciendo a esta segunda categoría hay una pregunta típicamente sociológica: ¿cómo actúa lo social sobre los individuos? ¿Cómo éstos informan sus conductas y prácticas? ¿Y qué es lo que hace que esta acción de lo social no sea jamás una completa 
determinación? Obsérvese que lo social está siempre pensado, en Bourdieu, bajo las categorías, algo antagónicas, por un lado, de necesidad, de restricción, de obligación y de imposición de reglas legítimas y generalizadas, y por el otro, de lo arbitrario y también de la indeterminación, de lo indefinido y lo difuso (Gautier, 2012).

Para resolver estas cuestiones, Bourdieu inventa una cierta cantidad de dualidades, mientras toma prestado un vocabulario del estructuralismo. Entre las palabras clave figuran las de objetivación e incorporación. No se trata de objetivación como operación cognitiva, sino como transformación en realidad objetiva (sobre todo la de los cuerpos y las instituciones). Incorporación significa que las estructuras, las tendencias y las necesidades objetivas del mundo están presentes en los cuerpos socializados, y sobre todo en los esquemas cognitivos o las estructuras mentales de los individuos. Los habitus son así la forma incorporada de las estructuras del mundo social ("el orden social se inscribe en los cuerpos”, - Bourdieu, 1997, p.168).

En las bases de la teoría del habitus existen, al menos, las tres dualidades siguientes. La primera es la dualidad de la objetivación de la historia: hay “dos objetivaciones de la historia”, una en los cuerpos individuales (esto es precisamente el habitus: los habitus son estructuras objetivas engendradas por la historia), otra en las instituciones. El encuentro entre lo social y lo individual es “el encuentro de dos historias” (Bourdieu, 1997, p.179). Producto de la historia, el habitus produce historia “de acuerdo con los esquemas engendrados por la historia” (Bourdieu, 1980, p.91). De esto deriva una segunda dualidad, la de los "estados objetivos del mundo social”: por un lado, el orden social es objetivado como conjunto estructurado de disposiciones o esquemas; por el otro, es objetivado en un campo social y en campos específicos estructurados de una cierta manera, es decir en un conjunto de instituciones, de reglas, posiciones y juegos más o menos formalizados. Por último, hay una dualidad de la formación y de la aplicación de las disposiciones: el habitus pone en relación, por un lado, las condiciones sociales de su formación, por ende los contextos de socialización, a través de los "condicionamientos asociados a una clase particular de condiciones de existencia”; por el otro, las condiciones sociales, o situaciones, en las cuales es puesto en práctica.

\section{Acuerdo y ajuste}

Las ideas de ajuste y acuerdo espontáneos están ligadas a estas dualidades. "Las disposiciones interiores, interiorización de la exterioridad, permiten que las fuerzas exteriores se ejecuten, pero según la lógica específica de los organismos en los cuales éstas son incorporadas, es decir de manera duradera, sistemática y no mecánica” (Bourdieu, 1980, p.92). Hay un ajuste del exterior y del interior, una conformidad de las disposiciones individuales con las propiedades objetivas de un campo, un "ajuste de las disposiciones a la posición”, un ajuste de las expectativas a las oportunidades, o un ajuste del habitus a las exigencias inmanentes de un juego social (Bourdieu, 1987, p.21). En resumen, el sistema de las disposiciones individuales acuerda con las regularidades del mundo natural y social. Es lo que permite que las estrategias producidas por el habitus sean “objetivamente amoldadas a la situación” (Bourdieu, 1987, p.21) o también “inmediatamente amoldadas” al orden social. Pero, por otra parte, el desacuerdo también es posible, más aun cuando las condiciones de actualización del habitus son diferentes de las que lo formaron. El habitus puede tornarse obsoleto por el cambio social, o no estar adaptado a una nueva posición social. 
Hay también un acuerdo espontáneo de los habitus individuales entre sí, porque son isomorfos. Bourdieu atribuye en efecto un status transindividual al habitus: las disposiciones son supraindividuales o colectivas (Bourdieu, 1997, p.186). Es lo que le permite explicar la intersubjetividad por el habitus:

Es el lugar de las solidaridades duraderas, de las fidelidades incoercibles porque basadas en leyes y lazos incorporados, los de la camaradería (...), adhesión visceral de un cuerpo socializado al cuerpo social que lo hizo y con el cual hace cuerpo. De este modo, es el fundamento de una colusión implícita entre todos los agentes que son el producto de condiciones y de condicionamientos similares, y también de una experiencia práctica de la trascendencia del grupo, de sus maneras de ser y de hacer, con la que cada cual encuentra en la conducta de todos sus pares, la ratificación y la legitimación («se hace») de su propia conducta que, a su vez, ratifica y, en el caso contrario, rectifica la conducta de los demás. Acuerdo inmediato en los modos de juzgar y de accionar, que no supone ni la comunicación de las conciencias ni, menos aún, la decisión contractual, esta collusio funda una intercomprensión práctica... (Bourdieu, 1997, p.173).

Los habitus, “orquestados entre sí”, producen "conjuntos de acciones que, fuera de todo complot y de toda concertación voluntaria, acuerdan groseramente entre sí” (Bourdieu, 1997, p.174).

Ya se dijo, Bourdieu utiliza indistintamente disposición y esquema para definir el habitus, e identifica más o menos esquema a estructura. Los habitus son "esquemas adquiridos funcionando en el estado práctico como categorías de percepción y de apreciación o como principios de clasificación, a la vez que como principios organizadores de la acción” (Bourdieu, 1987, p.24). Pero la idea de esquema, abundantemente usada tanto en psicología como en filosofía, no es menos enigmática que la de disposición. Como la disposición, el esquema se forma a espaldas del agente. Sus trazos característicos son la abstracción, la generalidad, la potencialidad y su carácter extrapolable. El esquema es abstracto en la medida que no tiene existencia material concreta. Parece poseer una suerte de vida autónoma. Es también algo general y extrapolable. Es, por último, como la disposición, algo "potencial”, en la medida que contiene potencialidades que pueden actualizarse. Quizá sería posible disipar un poco el misterio concibiendo el esquema no como una estructura sino como un método de operación, como una técnica para organizar una diversidad dinámica, o como "una regla de construcción para los objetos de una cierta especie o de un cierto género" (Chambon, 1974, p.254). Si también atribuimos esquemas al medio ambiente, ya sean procesos naturales, objetos o máquinas, podemos hablar de esquemas operatorios o de principios de constitución y de funcionamiento (Simondon, 1969).

\section{La psicología funcional de Dewey}

El científico y el filósofo, como el carpintero, el médico y el político, conocen con sus hábitos y no con su "conciencia”. Esta última es un resultado, no una fuente. Su aparición marca una conexión particularmente delicada entre hábitos altamente organizados e impulsos desorganizados (Dewey, 1922, pp. 182-182) 
La concepción de Dewey se quiere "funcional” (en un sentido que voy a detallar) o "transaccional”. Su análisis del hábito está expuesto principalmente en un libro de 1922, Human Nature and Conduct, que retoma una serie de conferencias dadas en 1918 (véase el subtítulo del volumen: Une introduction à la psychologie sociale). Es, a mi entender, más radical que la de Bourdieu. Bourdieu sostiene que el habitus es el "producto de la incorporación de la necesidad objetiva”, que es “necesidad hecha virtud” (Bourdieu, 1987, p. 21). Es una idea que parece bastante cercana a la de Dewey, según la cual los hábitos incorporan condiciones objetivas. Sin embargo, Dewey no entiende necesariamente incorporación en un sentido literal (cosa hecha cuerpo). De hecho, va más allá de un razonamiento en términos de ajuste al entorno, o de acuerdo entre las disposiciones y el entorno. Tiene una concepción mucho más “funcional” y más operacional de la incorporación de las condiciones objetivas en el hábito. Por un lado, el entorno también está configurado por hábitos; por otro, el esquema predominante no es el del acuerdo, como en Bourdieu, sino el de la co-operación en el sentido de una operación común, repartida en el organismo y el entorno que forman un solo sistema. La co-operación se basa en una integración de energías provenientes de estas dos fuentes.

Tres citas:

Una psicología basada en los hábitos (y en los instintos que se transforman en elementos de los hábitos cuando se acciona sobre ellos) se centrará, al contrario [de la psicología de la conciencia], en las condiciones objetivas en las cuales los hábitos son formados y en las cuales operan (Dewey, 1922, p.86).

El hábito es el motor de la acción humana y los hábitos están en gran parte formados bajo la influencia de las costumbres de un grupo (...). La influencia del hábito es decisiva porque toda acción específicamente humana debe ser aprendida, y hasta el corazón, la sangre y el nervio del aprendizaje son la creación de hábitos. Los hábitos nos unen a modos de acción ordenados y establecidos porque generan una comodidad, habilidad e interés por las cosas a las cuales nos hemos acostumbrado; generan el temor de tomar otros caminos y nos perjudican cuando se trata de hacerlo. El hábito no excluye el uso del pensamiento, pero determina los canales en los cuales opera. La reflexión es segregada en los intersticios entre los hábitos (Dewey, 2010, pp.255-6) ${ }^{\underline{3}}$.

Para cambiar el carácter activo o la voluntad de un otro distinto a nosotros, hay que accionar sobre las condiciones objetivas que entran en sus hábitos (...). No cambiamos los hábitos directamente. Eso sería magia. Los cambiamos indirectamente, modificando las situaciones... (Dewey, 1922, pp.19-20).

Estas citas tienen sin duda una resonancia bourdieusiana, aunque fueron escritas 50 años antes de Esquisse d'une théorie de la pratique (Bourdieu, 1972).

Ya lo dije, Dewey, al igual que Peirce, no habla de habitus sino de habit. Hay que observar también que en la tradición fenomenológica, en la cual Bourdieu se inspira en parte, Merleau-Ponty hablaba más de hábito que de habitus (aunque también use ese término algunas veces). Para este último, el hábito permite comprender la intencionalidad motriz. Su definición es cercana a la de Dewey (el 
hábito como «arte» o savoir-faire): "Si el hábito no es ni un conocimiento ni un automatismo, ¿qué es entonces? Se trata de un saber que está en las manos, que sólo se da en el esfuerzo corporal y que no puede traducirse en una designación objetiva” (Merleau Ponty, 1945, p.168). "Es el cuerpo el que entiende en la adquisición del hábito” (Merleau Ponty, 1945, p.169) y comprender es captar significaciones motrices a través del cuerpo motor. Para ilustrar la adquisición de hábitos, MerleauPonty alude a la señora que integró la altura de la pluma de su sombrero (al entrar en el subte, por ejemplo), al conductor que se acostumbró a las dimensiones de su auto, al ciego que percibe su entorno a través de su bastón, al organista que se instala fácilmente delante de un nuevo órgano que no le es familiar, a la dactilógrafa que integra el espacio del teclado de su máquina de escribir a su espacio corporal. Son argumentos que Bourdieu retomó y desarrolló hablando de "conocimiento por el cuerpo” (en Méditations pascaliennes sobre todo). Pero este último prefiere hablar de habitus antes que de hábito:

El habitus, como lo indica la palabra, es lo que se ha adquirido [...]. ¿Pero porqué no haber dicho hábito? El hábito es considerado espontáneamente como repetitivo, mecánico, automático, más reproductivo que productivo. Sin embargo, quiero insistir en la idea de que el habitus es algo potentemente generador (Bourdieu, 1980, p.134).

Dewey apunta sin embargo que utiliza el término habit en un sentido más amplio que el sentido usual. Dice que lo elige por sobre el de disposición, que puede inducir fácilmente al error, porque éste sugiere "algo latente, potencial, algo que requiere un estímulo positivo exterior para tornarse activo” (Dewey, 1922, p.41). El hábito no es exactamente de este orden: es actual y no potencial; es "una forma positiva de acción” más que una potencialidad. Voy a tratar de precisar este “actualismo” de Dewey. Este no excluye sin embargo que se hable de disposición, siempre y cuando que se entienda por ésta "una forma positiva de acción inhibida por una tendencia que la neutraliza” (Dewey, 1922, p.41). "Debemos entonces recordar que disposición significa predisposición, preparación para actuar abiertamente de una manera específica cada vez que se presenta la oportunidad, siendo esta oportunidad creada por la supresión de la presión ejercida por la predominancia de algún hábito manifiesto” (Dewey, 1922, p.41). Lejos estamos aquí de la concepción de la disposición como ley de comportamiento (Bourdieu, 1998), o hasta como mecanismo o maquinaria "montado para reaccionar de determinadas maneras" 4 (Wittgenstein, citado en Bouveresse, 1995, p.594).

Dewey prefiere, en vez de la categoría de disposición, las de arte, de capacidad, de habilidad, de destreza y de dominio de técnicas, siempre y cuando no se olvide que todo eso implica "materiales objetivos":

Los hábitos son artes [también podemos traducir art como savoir-faire]. Implican la habilidad [skill] de los órganos sensoriales y motores, la destreza o pericia, así como materiales objetivos. Asimilan energías objetivas (...). Requieren orden, disciplina y manifiestan una técnica. Tienen un comienzo, una mitad y un final [en el sentido de que se pueden adquirir, desarrollar y perder] (...). Nos reiríamos de alguien que dijera que sabe tallar piedras, pero que pretendiera que su savoir-faire está encerrado en él mismo y que no depende en absoluto del soporte que encuentra en los objetos y de la asistencia de sus herramientas (Dewey, 1922, p.15). 
Sabe de hecho, y de manera práctica, cómo se comporta tal tipo de piedra, o tal o cual parte de la piedra cuando se la golpea, cómo reaccionan sus herramientas a tal tipo de piedra o tal tipo de golpe, con qué ángulo debe orientar el golpe de la herramienta, cuándo cambiar de herramienta, etc. En términos que no son los de Dewey sino de G. Simondon (1969, p.77), podemos decir que su cuerpo “arrutinado" (un término del francés antiguo) $\frac{5}{5}$ se tornó el “medio asociado" de objetos y herramientas. La formación del hábito se hace de este modo en una participación senso-motriz en las cosas y acontecimientos del entorno, una participación que tiene una dimensión cognitiva, afectiva y práctica, a la vez.

\section{Hábito, rutina, repetición}

Tenemos la tendencia, indica Dewey, a considerar los hábitos como malos, como serviles a surcos trazados que tomamos espontáneamente, repetidamente y sin reflexión. Pero no hay que identificar el hábito a la rutina, porque este último término resalta el aspecto de repetición automática o mecánica de actos específicos realizados sin pensar (que Dewey llama “el hábito sin inteligencia”):

La repetición no es de ningún modo la esencia del hábito. (...) La esencia del hábito es una predisposición adquirida a maneras de actuar (ways) o a modos de respuesta, y no a actos particulares, excepto en la medida en la que éstos, bajo ciertas condiciones, expresan una manera de comportarse (Dewey, 1922, p.42).

“Adquirida” significa formada en una actividad o una experiencia anterior. Podemos también considerar el hábito como una "sensibilidad o una accesibilidad particular a ciertas clases de estímulos, predilecciones y aversiones permanentes más que una simple recurrencia de actos específicos” (Dewey, 1922, p.42). Cuando se convierte en una rutina mecánica, una huella, el hábito torna inútil o imposible el pensamiento; restringe el pensamiento: “Estímulo y respuesta están mecánicamente ligados en una cadena sin fallas. Cada acto sucesivo es fácilmente suscitado por el que lo precede, y nos empuja automáticamente hacia el siguiente en una serie predeterminada” (Dewey, 1922, p.173). Pero las rutinas pueden volverse inoperantes por circunstancias imprevistas.

A decir verdad, la idea misma de rutina es también ambivalente. Los automatismos adquiridos no son necesariamente huellas que tomamos de manera repetitiva: tornan también posible la acción inteligente. Podemos definir en efecto una rutina como "lo que, sin acaparar la atención ni la energía, brinda una base constante para la liberación de valores de la vida intelectual, de la vida estética y de la vida en compañía de los demás” (Dewey, 1935, p.89)ํㅡ. La excelencia en la práctica de un oficio o de un arte no puede ser alcanzada sin la formación de rutinas. En sentido propio, la rutina resulta de la apertura de un camino. Un obrero "bien arrutinado", como se decía en el siglo XVI, es el que es experimentado, “ducho” en el ejercicio de su arte. Adquirida a fuerza de ejercicio y únicamente perdurando por su práctica continua (sin práctica, se pierde), la rutina es lo que le permite estar plenamente en su tarea, concentrarse en lo que hace, sin representarse objetivos y ahorrarse reflexión y razonamiento, deliberación y planificación (Vérin, 1998). No se debe entonces tratar la rutina solamente como un hábito no inteligente y oponer la rutina al arte, como lo hace a veces Dewey (Dewey, 1922, p.77). 
Dewey critica el lugar usualmente otorgado a la repetición en la formación de los hábitos. Para que haya repetición, es necesaria una uniformización de las condiciones circundantes:

Decir que los hábitos se forman por simple repetición, es poner el carro delante de los bueyes. La capacidad de repetir es el resultado de la formación de un hábito gracias a las nuevas disposiciones orgánicas que produce la consumación de un acto hasta el final; esta modificación equivale a dar una dirección determinada a las acciones futuras (Dewey, 1993, p.91).

Lo que cuenta en la formación de un hábito es menos la repetición que "la institución de una interacción y de una integración efectivas de las energías del organismo y del entornoconsumación, final y coronación de actividades de exploración y de búsqueda” (Dewey, 1993, p.90, resaltado por el autor). Un hábito tiene que ser flexible y readaptable, sino, no podrá adaptarse a un cambio posterior de las condiciones circundantes.

Aprender es formar nuevos hábitos de acción, y formar nuevos hábitos es modificar las necesidades y renovar las relaciones con el mundo circundante, y esto porque "cada hábito exige condiciones apropiadas para su ejercicio” (Dewey, 2012, p.281). El que formó nuevos hábitos no sólo se transformó a sí mismo, también adquirió un nuevo entorno.

«Cuando los hábitos son numerosos y complejos, como es el caso en el hombre, descubrir las [condiciones apropiadas para el ejercicio del hábito] implica emprender una búsqueda y experimentaciones; el organismo es forzado para provocar variaciones, y se expone al error y a la decepción. Puede parecer paradójico que el aumento de la capacidad de formar hábitos signifique que aumentan también la sensibilidad, la perceptibilidad, la receptividad. De este modo, si concebimos los hábitos como cualquier otra rutina, la capacidad de adquirir muchas rutinas variadas denota una alta sensibilidad y receptividad. Así, un viejo hábito, una rutina congelada, si exageramos, forma un obstáculo para el proceso de formación de nuevos hábitos, mientras que la tendencia a formar nuevos hábitos va en dirección contraria a algún antiguo hábito» (Dewey, 2012, p.260, resaltado por el autor).

Hay que observar sin embargo que la formación de hábitos personales siempre se da "en condiciones fijadas por costumbres que las preceden”, a través de usos y costumbres y a través de hábitos colectivos establecidos, es decir instituciones (Dewey, 1922, p.58), lo que explica la persistencia de las costumbres: "Siempre y en todo lugar son las costumbres las que otorgan sus patrones a las actividades personales” (Dewey, 1922., p.75).

\section{El modelo ecológico de Dewey}

Me gustaría ahora explicitar los caracteres principales del hábito en Dewey: su integración del entorno y no solamente en el entorno, su carácter activo, su carácter organizado y su omnipresencia.

Ya dije que el modelo de Dewey era biológico y ecológico. ¿Qué quiere decir esto? Una función biológica como la respiración o la digestión está basada en una cooperación del organismo y del entorno: el cuerpo humano y sus órganos no son suficientes, también son necesarios el aire, la comida, y no cualquier cosa puede servir de comida. En resumen, es necesario un aporte de energías 
externas. Es por eso que podemos decir que estas funciones son cosas hechas por el cuerpo humano con la ayuda del entorno, como también que son "cosas hechas por el entorno via las estructuras orgánicas” del cuerpo humano (Dewey, 1922, p.14). No hay sólo interacción del organismo y del entorno, sino transacción. Transacción significa operaciones conjuntas, distribuidas y articuladas en un sistema integrado, como también composición ordenada de energías externas e internas. Una parte de estas operaciones es efectuada en y a través de las cosas, según lo que podemos llamar funcionamientos, otra parte lo es en y a través del organismo en función de sus capacidades biológicas y de su equipamiento cultural. Dewey también tiene presente la noción matemática de función, que es relacional: una función es una relación que, con el valor de una variable independiente “ $x$ ”, hace corresponder un valor “ $y$ ” $(y=f(x))$.

Dewey integra así en el hábito el dinamismo de las cosas, es decir su energía y su régimen operacional (el entorno no es inerte, cambia en permanencia y es un operador): no sólo el entorno es necesariamente interno al hábito, sino que las actividades vitales también son llevadas a cabo indistintamente por el entorno via hábitos, y por hábitos via el entorno. En las funciones vitales, el organismo usa al entorno y éste usa al organismo. El entorno social de usos, costumbres e instituciones no constituye una excepción; son también fuentes de energía y fuerzas operacionales (y no únicamente en el sentido de coerciones u obligaciones):

Cada institución aportó, en su desarrollo, obligaciones, expectativas, reglas, patrones. No son simples adornos de fuerzas los que los produjeron. Son fuerzas adicionales. Reconstruyen. Abren nuevos caminos para el esfuerzo e imponen nuevas tareas. En resumen, son la civilización, la cultura, la moralidad (Dewey, 1922, p.80).

Y queda en Dewey plantear la pregunta por la autoridad de estas ideas, obligaciones, reglas, patrones: "Su autoridad es la de la vida”, simplemente porque si se quiere vivir, no se puede hacer otra cosa que "vivir una vida en la que todas estas cosas forman la sustancia. La única pregunta que tiene sentido es: cómo vamos a utilizar estas cosas y como seremos usados por ellas” $\underline{7}$ (Dewey, 1922, p.81). La perspectiva de Dewey es la de tornar las costumbres e instituciones más inteligentes, transformarlas a través de la crítica y de la investigación.

Si las transacciones entre un organismo y un entorno ponen así en juego un sistema integrado de energías y una “conjunción coordenada” de operaciones, tiene sentido decir que hay "hábitos despersonalizados del entorno” (Dewey, 1922, p.22), que son más que las regularidades de comportamiento de las cosas, es decir modos operacionales de los objetos. Un hábito no es entonces la "posesión privada de una persona”. En tanto manera general de actuar, implicado en actividades o prácticas instituidas, e incorporando materiales objetivos, está tanto en el entorno como en el organismo, si entendemos que está repartido ( $c f$. la cita acerca del tallador de piedra). Es un punto importante aunque algo sorprendente. Pero, por otro lado, los factores personales cuentan, evidentemente, en el hábito. Por ello todo el mundo no tiene los mismos gustos. Pero los gustos no se forman en una conciencia cerrada sobre sí misma. Se forman en interacciones con el entorno, y en este sentido, tienen un soporte objetivo.

De un modo más general, Dewey dirige la atención a los sesgos que introduce el discurso cuando vincula los actos y actividades a un self construido. Por una parte, no hay self construido, sino self 
que se descubre, se hace y rehace actuando. Por otra, detrás de las actividades hay otra cosa que un self ya plenamente constituido: "Hay actitudes, hábitos e impulsos complejos, inestables, que se oponen unos a otros; progresivamente logran componer unos con otros, y adoptan una cierta coherencia de configuración” (Dewey, 1922, p.138).

Dewey no deja de repetir que un organismo no vive solo en un entorno sino también, y más fundamentalmente, a través de un entorno. Es por eso que hay que partir de la idea de que los hábitos son "maneras de usar y de incorporar el entorno, en los cuales este último tiene tanto peso como el organismo" (Dewey, 1922, p.15). Es la razón por la cual son más que ajustes a un entorno: "Los hábitos incorporan en ellos un entorno. Son ajustes del entorno, y no simplemente $a$ él” (Dewey, 1922, p.153). Es una idea que exige ser explicada.

El entorno es interno al organismo y el organismo interno al entorno, sobre todo por el hecho de su constitución física e intelectual: una vaca (herbívora) no tiene el mismo entorno que el perro (carnívoro) que la vigila, o el pájaro (insectívoro) que encuentra qué comer en su bosta; tratándose de humanos, un médico no tiene el mismo entorno que un mecánico: “Un organismo vivo y sus procesos vitales implican un mundo o una naturaleza que es temporalmente y espacialmente «exterior» a él, pero «interna» a sus funciones” (Dewey, 2012, p.278), como lo demuestran las funciones biológicas. Es precisamente esta participación en las actividades vitales lo que define un entorno, al igual que son las “conexiones activas” con un entorno las que definen un organismo:

Hay ciertamente un mundo natural que existe independientemente del organismo, pero ese mundo sólo es entorno si entra directamente e indirectamente en las funciones vitales. El organismo forma él mismo parte del vasto mundo natural y sólo existe como organismo en las conexiones activas con su entorno. La integración es más fundamental que la distinción designada por la interacción del organismo y del entorno. Esta última designa la desintegración parcial de una integración anterior... (Dewey, 1993, p.92).

La dualidad organismo/entorno es entonces segunda en relación a lo que existe realmente, es decir un sistema integrado en el cual tiene lugar una composición de energías y una "conjunción coordenada" de operaciones. En consecuencia, un organismo es "una criatura engendrada por el intermediario de una unión conjuntiva”, y "comporta en el interior de su propia estructura los órganos y las marcas de su conexión íntima con otras criaturas” (humanas y no humanas) (Dewey, 1993, p.92). Es de hecho por esto que Dewey preferirá el término transacción al de interacción.

En un sentido, Dewey pone los hábitos en el mismo plano que la constitución orgánica del organismo, porque, como esta última, los hábitos son las mediaciones de la operatividad del entorno en la efectuación de las actividades vitales. Hay funciones sociales al igual que funciones fisiológicas y los hábitos, que son siempre sociales, son el soporte de ellas. En particular informan al entorno, que se organiza según su esquema y, a su vez, requieren de este entorno para ejercerse. Una tribu de cazadores no tiene la misma configuración de hábitos que informan sobre todas sus actividades, también las artísticas, que una tribu de agricultores sedentarios. Una y otra viven en mundos diferentes. Para la primera, por ejemplo, las plantas son lugares en donde los animales pueden esconderse, y la tierra no es considerada como un medio de producción de cosechas. Y ella se encargará de actuar el drama de la caza en todas sus actividades (el ejemplo es del mismo Dewey 
- cf. Garreta, 2002 - ).

Dewey saca una conclusión importante de esta imbricación del hábito y del entorno: es vano querer cambiar los hábitos si no se cambia el entorno, y sobre todo las costumbres, los "arreglos sociales" y las instituciones, que son hábitos ellas también ("La fuerza que más influye en las disposiciones y las actitudes de los individuos es el medio (medium) social en el cual estos viven” -Dewey, 1935, p.91-). Y, recíprocamente, no se puede cambiar el entorno sin cambiar los hábitos adquiridos.

\section{La primacía de los hábitos sobre el instinto y el impulso}

Pero para cambiar los hábitos adquiridos, hay que "redirigir los impulsos (impulses)” (Dewey, 1922, p.126). Dewey otorga un lugar importante a los impulsos, término que prefiere al de instinto (Dewey, 1922, p.105, nota). En un sentido, son los impulsos los que están temporalmente primero en la conducta, pero totalmente desorganizados. Sólo adquieren significado en interacción con un medio social, por lo tanto via hábitos. Son entonces los hábitos los que importan, no los instintos o los impulsos; éstos dependen de hecho de los hábitos: "La posición del impulso en la conducta es una posición intermediaria” (Dewey, 1922, p.169), porque está contenido y dotado de sentido a través de los hábitos. Sin embargo, sigue siendo fuente de creatividad y de renovación:

El correlato moral del impulso liberado no es la actividad inmediata, sino la reflexión acerca del modo en que se puede usar el impulso para renovar las predisposiciones y reorganizar los hábitos. (...) Hay un impulso para suscitar el pensamiento, incitar a la reflexión y vivificar la creencia. Pero sólo el pensamiento (...) convierte el impulso en un arte que vive en los objetos. El pensamiento nace como el mellizo del impulso cada vez que el hábito es entorpecido (Dewey, 1922 pp.170-71).

El impulso también tiene su importancia para el cambio social, porque puede ser un puntapié para la reforma; contribuye a reorganizar, a reajustar o a formar nuevos hábitos: los impulsos son "los ejes sobre los cuales gira la reorganización de las actividades, son productores de desvíos, dan nuevas direcciones a viejos hábitos y cambian su calidad” (Dewey, 1922, p.93). La educación misma se nutre de una reserva considerable de impulsos no usados: "El impulso es una fuente, una fuente indispensable, de liberación; pero lo es sólo si es usado para dar pertinencia y frescura a los hábitos” (Dewey, 1922, p.105).

Dewey hubiese sido entonces escéptico ante al poder que Bourdieu le otorga a la toma de conciencia por el autoanálisis, aunque haya argumentado que la disposición para criticar, para reflexionar y para investigar, en tanto costumbre socio-históricamente establecida, sea "capaz de ejercer la influencia más revolucionaria sobre otras costumbres” (Dewey, 1922, p.78). Cito a Bourdieu: El habitus puede también ser transformado a través del socio-análisis, la toma de conciencia que permite al individuo influir en sus disposiciones. Pero la posibilidad y la eficacia de esta suerte de auto-análisis [sic] están en sí mismas determinadas en parte por la estructura original del habitus en cuestión, en parte por las condiciones objetivas en las que se produce esta toma de conciencia (Bourdieu, 1992, p.239).

Dewey cree más en la comunicación que en el autoanálisis. En los organismos superiores, lo hecho está condicionado por las consecuencias de sus actividades anteriores, porque éstas forman hábitos. 
Es lo que permite que un entorno espacio-temporal distante entre en la conducta. Pero también ir más allá de la simple repetición, y esto gracias a la comunicación entre los hábitos.

En ausencia de comunicación, la formación de los hábitos utiliza rutinas (wearsgrooves); el comportamiento es mantenido en canales establecidos por los comportamientos anteriores. En esta medida, la tendencia es la regularidad monótona. La operación misma del aprendizaje se fija límites, de manera que el aprendizaje posterior se torna más difícil. Pero todo esto es cierto para un hábito, un hábito aislado, un hábito que no comunica. La comunicación no sólo aumenta el número y la diversidad de los hábitos, sino que también tiende a ligarlos sutilmente unos a otros y, eventualmente, a subordinar la formación de hábitos en un caso particular al hábito de reconocer que nuevos modos de asociación exigirán un nuevo uso [de este hábito]. De este modo, el hábito es formado en vistas a futuros cambios posibles y no se fija tan fácilmente (Dewey, 2012, p.259).

Cuanto más numerosos y flexibles son los hábitos, más puede la percepción discriminar finamente, y más fino es lo que es evocado por la imaginación. Dewey no dice más acerca de lo que puede ser esta comunicación entre los hábitos.

\section{Los hábitos como savoir-faire}

Arribo ahora a los demás caracteres del hábito tal y como lo concibe Dewey, en primer lugar por sus dimensiones activas y organizadas. Ya lo he dicho, Dewey conceptualiza el hábito en términos dinámicos de energía, de actividad, de operación, de arte o de savoir-faire, más que de disposición: los hábitos son maneras de actuar adquiridas por la experiencia (opuestas a las actividades nativas o del instinto), siendo éstas maneras de actuar generales y organizadas (Dewey, 1922, p.89). Cuando son formuladas, se transforman en reglas, leyes o principios de acción. Así es como Dewey define los caracteres activo y organizado del hábito:

“Podemos concebir los hábitos como medios, que esperan, como las herramientas de una caja, ser usados por una decisión consciente. Pero son más que eso. Son medios activos, medios que se proyectan a sí mismos, maneras de actuar energéticas y dominantes. (...) [Las herramientas] sólo son medios cuando son empleadas en cualquier hacer real. Sino, sólo son (...) medios potenciales. Sólo son medios reales cuando convergen con el ojo, el brazo y la mano en cualquier operación específica. Y el ojo, el brazo y la mano sólo son herramientas propiamente dichas, de modo correspondiente, cuando están en operación activa. Y cuando están en acción, cooperan con materiales y energías externas. Sin este soporte que los excede, la mirada del ojo está vacía y la mano se mueve con torpeza. Sólo son medios cuando entran en una organización con cosas que llevan a cabo por sí mismas resultados definidos. Estas organizaciones son hábitos (Dewey, 1922, pp.25-26).

Así, Dewey insiste en la importancia decisiva de la operación activa a la vez que en la "conjunción coordinada" de los materiales externos y de los órganos corporales cuando son "empleados en un hacer real”. En resumen, lo que distingue al hábito de la disposición es que éste sólo es tal cuando está en “operación activa” en una co-operación con el entorno. Además, es su carácter organizado lo que hace que el hábito no sea una simple tendencia o impulso: "Contiene en sí mismo una cierta planificación o sistematización de pequeños elementos de acción” (Dewey, 1922, pp.40-41). 
Otro aspecto de la organización de los hábitos es su articulación entre ellos. Efectivamente, los hábitos son plurales y ligados los unos a los otros; se afectan mutuamente y se interpenetran, creando una continuidad entre ellos; operan en su diversidad en todo acto, modificándose y reforzándose mutuamente. De este modo, para escribir una carta a mano hay que saber escribir; también se necesita papel, birome y un destinatario; es necesario tanto una serie de hábitos compartidos como la escritura, la lectura, la correspondencia como un sistema postal. Es esta interpenetración de hábitos lo que define un carácter (Dewey, 1922, p.38). Pero por otro lado, los hábitos pueden entorpecerse, contradecirse, entrar en conflicto. Un conflicto de hábitos desencadena emociones y crea un espacio para la reflexión porque libera impulsos que exigirán una modificación de los hábitos.

Los hábitos no intervienen solamente en las actividades corporales o manuales. La formación de las ideas y su puesta en práctica dependen también del hábito: "Una idea sólo adquiere forma y coherencia cuando se respalda en un hábito” (Dewey, 1922, p.30), y esto porque los actos preceden al pensamiento. Funciona igual con las sensaciones (Dewey, 1922, p.31). Separar el hábito del pensamiento es un error, sobre todo porque un pensamiento que no se instala en el interior de los hábitos de acción corrientes está desprovisto de medios de ejecución, y por ende de criterios (Dewey, 1922, p.67).

\section{La omnipresencia de los hábitos}

No hay entonces conducta inteligente sin hábito. Para Dewey, no es el espíritu o la conciencia lo que está en el origen de la conducta inteligente, sino los hábitos; conocemos y juzgamos con nuestros hábitos; son "los únicos agentes de la observación, del recuerdo, de la anticipación y del juicio” (Dewey, 1922, p.176); “Son los hábitos concretos los que hacen todo el trabajo de percepción, de reconocimiento, de imaginación, de rememoración, de juicio, de concepción y de razonamiento" (Dewey, 1922, p.177). Cuanto más numerosos son, más amplio es el "campo de la observación y de la predicción posibles”; “más flexibles son, más fina es la percepción en sus distinciones y más delicado lo que evoca la imaginación” (Dewey, 1922. pp.175-6). Los hábitos hacen el "trabajo práctico" de ajuste rápido y preciso al entorno -pero no se trata de conocimiento, en el sentido de «knowledge about» o de «knowledge that». Es también porque tenemos hábitos que podemos estar en una situación determinada: "Los hábitos entran en la constitución de la situación, están en ella y son de ella, no le son externos” (Dewey, 1916 -citado en Garreta, 2002, p.152).

Pero, por otro lado, no son los hábitos los que suscitan la reflexión, el análisis o la investigación, porque son ellos mismos "demasiado organizados, demasiado apremiantes y demasiado determinados como para necesitar que la investigación o la imaginación sigan su curso”, o también adaptados con demasiada precisión al entorno como para analizarlo (Dewey, 1922, p.177). Se necesita un traspié, algo que falle, un problema, una perturbación, un obstáculo en el ajuste al entorno, que requiera un reajuste y por ende un cambio en la actividad, una redistribución de sus elementos para provocar el pensamiento propiamente dicho, así como la emoción. Cuando esto se produce, hay perturbación, confusión, incertidumbre. Lo que sucede entonces es el desencadenamiento de un impulso, que tiende a reorientar la actividad desorganizada en una nueva dirección (un impulso arranca alguna cosa a las apuradas), y que suscita una investigación; como 
son espontáneos y no organizados, los impulsos pueden ser contradictorios; pueden bloquear los hábitos o entrar en conflicto con ellos.

El impulso define la mirada, la búsqueda, la investigación. Es, formulado en términos lógicos, el movimiento en lo desconocido, no en lo desconocido en general, sino en este desconocido particular que, cuando nos topamos con él, restaura una acción ordenada, unificada. Durante esta búsqueda, los hábitos antiguos proporcionan el contenido, lo definido y lo reconocible. Esto comienza por un presentimiento difuso acerca de eso hacia donde nos dirigimos. A medida que los hábitos organizados están precisamente desplegados y focalizados, la situación confusa toma forma, es «esclarecida» (esto es la función esencial de la inteligencia). Los procesos devienen objetos. Sin hábito sólo hay irritación y duda confusa [porque los impulsos no tienen organización]. Pero si sólo hay hábito, sólo hay repetición maquinal, repetición que copia viejos actos. A partir del momento en que hay conflicto de hábitos y desencadenamiento de impulsos, hay búsqueda consciente (Dewey, 1922, p.180), además de distinción e identificación de objetos.

El hábito deweyano está así más cerca de una capacidad que de una disposición, para retomar una distinción apreciada por Wittgenstein quien, por diversas razones, criticaba vigorosamente la idea de disposición ( $c f$. Chauviré, 2002), sobre todo porque veía allí algo puramente hipotético, algo que evoca una maquinaria escondida generando performances reales, o también una "sombra de la realidad en donde estaría proyectado el conjunto de las performances” (Chauviré, 2002, p.34): “La disposición es una posibilidad y esta posibilidad, se la concibe ahora como sombra de la realidad" (Wittgenstein, citado por Chauviré, 2002). Una capacidad no es una posibilidad, sino el dominio de una técnica o de un método a partir de un aprendizaje, y una técnica tiene un componente normativo: "El concepto de capacidad es normativo, implica una referencia a la nociones de éxito o de fracaso, de corrección o incorrección de una performance, así como la indicación posible de una regla en tanto razón de la acción” (Chauviré, 2002, p.35). Dewey sin embargo no rechaza completamente la idea de un mecanismo: Todos los hábitos implican una mecanización. El hábito es imposible sin establecimiento de un mecanismo de acción, implantado psicológicamente, que opera «espontáneamente», automáticamente, cada vez que la señal es lanzada” (Dewey, 1922, p.70). Pero hay más en el hábito que el automatismo tonto y la rutina mecánica. El músico en la cima de su arte adquirió técnica y automatismos. Pero cuando toca, estos se fusionan con la emoción y el pensamiento, si no sus actuaciones se volverían mecánicas. Sus hábitos son inteligentes. Son susceptibles de ser mejorados, vueltos más flexibles y más sensibles, a través de la práctica.

\section{En conclusión}

Llegó el momento de repasar las diferencias entre la concepción de Bourdieu y la de Dewey -no retomo las afinidades y las similitudes, que son más evidentes. La diferencia principal tiene que ver con la manera de concebir la interiorización de la exterioridad. El modelo de Bourdieu sigue más ligado a una filosofía del sujeto que el de Dewey. En Bourdieu, la incorporación de las condiciones o estructuras objetivas se hace en un cuerpo individual, o en un sujeto capaz de "conocimiento por el cuerpo": "El habitus es una subjetividad socializada”ㅁ (Bourdieu, 1992, p.101). En Dewey, la interiorización del entorno es concebida más bien en términos de participación activa de las cosas en las funciones biológicas y más ampliamente en las actividades vitales (y no como formación de 
estructuras mentales), porque el entorno es fuente de energías y base de operaciones, operaciones que son funcionamientos. Desde este punto de vista, el modelo de Dewey es sin duda más circular que el de Bourdieu (lo hemos visto, las relaciones entre hábitos y entorno son recíprocas).

Una segunda diferencia importante tiene que ver con la elaboración de Dewey de una alternativa real al disposicionalismo, esquema explicativo que Bourdieu no rechaza. Para Dewey, el hábito se diferencia de la disposición en la medida en que es más "una forma positiva de acción” que una potencialidad, o dicho de otro modo, una organización estabilizada de operaciones conjuntas y articuladas del organismo y del entorno. Un hábito sólo es verdaderamente un hábito cuando está en operación activa, en una “conjunción coordenada” de operaciones y energías, estando éstas repartidas en el organismo y el entorno.

Una tercera diferencia tiene que ver con la concepción de la trascendencia de lo social. Bourdieu parece permanecer fiel a Durkheim en este punto, al que Merleau-Ponty reprochaba haber pensado mal esta trascendencia, sobre todo al haberla concebido en relación a los individuos, haber hecho de lo social una entidad positiva separada, un orden en sí exterior a los individuos, por ejemplo bajo la forma de una conciencia colectiva o de una fuerza sui generis (cf. Guchet, 2001). Dewey desconfía de este tipo de abstracción; considera más bien al entorno social como un medium (como medio -en el sentido de eso en donde la gente vive y lo que hay entre ella como "tejido conjuntivo", según Merleau-Ponty-), mediación y recurso, todo esto a la vez. Sabemos que Dewey fue influenciado no sólo por Darwin sino también por Hegel, y de una cierta manera este medium social no es nada menos que el espíritu objetivo hegeliano, es decir un medio cultural de tradiciones, de costumbres, de instituciones, prácticas y significados instituidos, métodos y técnicas establecidos, que tienen una cierta coherencia entre sí, y que son incorporados en los hábitos individuales. La invocación de una conciencia colectiva mística a la Durkheim le parece una explicación artificial. Podemos evitarla si partimos del hábito.

En cuarto lugar, en Dewey, el hábito es solamente una de variadas herramientas conceptuales para describir el sentido práctico o la lógica de la práctica (los otros medios son la crítica del arco reflejo, el tomar en cuenta la serialidad del comportamiento, la reconsideración de la dialéctica de los medios y los fines y una teoría de la formación de los valores - cf. Joas, 1999 -).

También podemos pensar que Dewey hubiese sido escéptico ante el patrón estructuralista de Bourdieu. No se trata de negar la existencia de estructuras, sino de otorgarles el lugar correcto. Para esto, sostiene Dewey, hay que razonar en términos funcionales y relacionales, y evitar hacer de la estructura una entidad o una sustancia. Una estructura sólo es estable y “estructurante” en relación a acontecimientos, procesos y cambios. Esto es lo que Dewey escribía en 1925, poco años después de su Human Nature and Conduct (1922),

llamar «estructura» a los acontecimientos más lentos que ofrecen ritmos más regulares y «procesos» a los acontecimientos más rápidos y más irregulares demuestra un sentido común práctico. Se expresa de este modo la función de uno con respecto al otro. (...) Toda estructura es una estructura de algo. Todo lo que se define como estructura es solo un carácter de los acontecimientos y no algo intrínseco y per se. Llamamos «estructura» a un conjunto de rasgos que cumplen una función de limitación con respecto a otros rasgos de los acontecimientos (Dewey, 2012, p.94-95). 
Por ejemplo, una casa tiene una estructura determinada, que es fija:

No es algo externo a lo que los cambios implicados en la construcción y el uso de la casa deban someterse. Se trata mejor dicho de un arreglo de acontecimientos cambiantes que hace que las propiedades que cambian lentamente limiten y dirijan una serie de cambios rápidos, y que le otorgue un orden que no tendrían de otro modo. Una estructura es una constancia de medios, de cosas usadas con miras a sus consecuencias, y no de cosas consideradas en ellas mismas o absolutamente. Una estructura es lo que torna posible una construcción, y no se puede ni descubrirla ni definirla independientemente de una construcción realizada, siendo esta construcción solamente un orden de cambios manifiesto. Cuando aislamos la estructura de los cambios cuya organización estable constituye, hacemos algo misterioso, metafísico en el sentido popular del término, una suerte de extrañeza fantasmal (Dewey, 2012, p. 94-95).

Cada una de las teorías presentadas tiene su interés, y su confrontación es estimulante. Finalmente Dewey no está menos convencido que Bourdieu de la determinación social de los comportamientos, así como del rol social de los hábitos. Pero no hay lugar en Dewey para una concepción durkheimiana de la sociedad, ni para un estructuralismo cualquiera, aunque este sea genético, fundamentalmente porque su teoría del hábito, y a través de ella, su teoría del comportamiento o de la conducta, están basadas en una filosofía del acontecimiento.

\section{Notas}

1 Texto escrito originalmente para una conferencia dictada en la Universidad de Buenos Aires el 13 de octubre de 2016, titulada: Bourdieu et le pragmatisme américain sur la créativité de l'habitude.

$\underline{2}$ Obsérvense las dualidades: estructuras sociales/estructuras mentales, estructuras objetivas/estructuras subjetivas, campos/individuos biológicos

$\underline{3}$ De aquí en adelante se deja constancia que, exceptuando Human Nature and Conduct (1922) y Liberalism and Social Action (1935), todos los textos de J.Dewey citados fueron tomados de traducciones francesas, posteriores a la versión original en inglés, tal como se señala en la Bibliografía.

4 Concepción que tampoco es la del habitus en Bourdieu

$\underline{5}$ Nota de la traductora: «routiné» en el texto original

$\underline{6}$ Dewey retoma aquí un argumento ya desarrollado por James - $c f$. Kilpinen, 2009 -

$\underline{7}$ Obsérvese el doble sentido, ya referido, del uso: usar las cosas, y ser usado, instrumentalizado, por ellas

$\underline{8}$ Dewey estaría de acuerdo si por subjetividad entendemos impulsos y no self

\section{Referencias bibliográficas}

Bourdieu, P. (1998). Savoir faire. Contribution à une théorie dispositionnelle de l'action. Paris: Seuil. 
Bourdieu, P. (1972). Esquisse d'une théorie de la pratique. Genève: Droz.

Bourdieu, P. (1980). Le sens pratique. Paris: Minuit.

Bourdieu, P. (1980). Questions de sociologie. Paris: Minuit.

Bourdieu, P. (1987). Choses dites. Paris: Minuit.

Bourdieu, P. (1992) Réponses. Paris: Seuil.

Bourdieu, P. (1997). Méditations pascaliennes. Paris: Seuil.

Bouveresse, J. (1995). Règles, dispositions et habitus (pp. 573-594). Critique, 579-580.

Chambon, R. (1974). Le monde comme perception et réalité, Paris: Vrin.

Chauviré, C. (2002). Dispositions ou capacités ? La philosophie sociale de Wittgenstein. En C. Chauviré \& A. Ogien (eds), La régularité. Habitude, disposition et savoir-faire dans l'explication de l'action (pp. 25-48). Paris: Editions de l’EHESS (Raisons pratiques 13).

Dewey, J. (1922). Human Nature and Conduct. An Introduction to Social psychology, New York, Henry Holt and Company.

Dewey, J. (1935). Liberalism and Social Action. New York: Prometheus Books.

Dewey, J. (1993). Logique. Théorie de l'enquête. Paris: PUF [1938].

Dewey, J. (2010). Le public et ses problèmes. Paris: Gallimard [1927].

Dewey, J. (2012). Expérience et Nature. Paris: Gallimard [1925].

Garreta, G. (2002). Une régularité sans répétition ? L’habitude comme schème dynamique. En C. Chauviré \& A. Ogien (eds). La régularité. Habitude, disposition et savoir-faire dans l'explication de l'action (pp. 137-160). Paris: Editions de l'EHESS (Raisons pratiques 13).

Gautier, C. (2012). La force du social. Paris: Cerf.

Guchet, X. (2001). Théorie du lien social, technologie et philosophie: Simondon lecteur de Merleau-Ponty (pp. 219-237). Les études philosophiques, 57 (2).

Haber, S. (2004). La sociologie française contemporaine devant le concept bourdieusien d'habitus (pp. 191-216). Alter. Revue de phénoménologie, 12.

Héran, F. (1987). La seconde nature de l'habitus. Tradition philosophique et sens commun dans le langage sociologique, Revue Française de Sociologie, 28 (3), p. 385-416.

James, W. (1890). The Principles of Psychology (vol. 1). New York: Henry Holt and Company.

Kilpinen, E. (2000). The Enormous Fly-Wheel of Society: Pragmatism's Habitual Conception of Action and Social Theory. Department of Sociology, University of Helsinki: Research Reports no. 235.

Kilpinen, E. (2009). The habitual conception of action and social theory (pp. 99-128). Semiotica, $173(1 / 4)$. 
Merleau Ponty, M. (1945). Phénoménologie de la perception. Paris: Gallimard.

Shusterman, R. (1995). Bourdieu et la philosophie anglo-américaine (pp. 595-609). Critique, 579580.

Simondon, G. (1969). Du mode d'existence des objets techniques, Paris: Aubier.

Vérin, H. (1998). La réduction en art et la science pratique au $\mathrm{XVI}^{\mathrm{e}}$ siècle, en R. Salais et al. (eds), Institutions et conventions (pp. 119-144) Paris: Editions de l'EHESS (Raisons pratiques 9). 